\title{
Mechanisms of Action and Targets of Nitric Oxide in the Oculomotor System
}

\author{
Bernardo Moreno-López, ${ }^{1}$ Carmen Estrada, ${ }^{1}$ and Miguel Escudero ${ }^{2}$ \\ 1Departamento de Fisiología, Facultad de Medicina, Universidad Autónoma de Madrid, 28029 Madrid, Spain, and Área \\ de Fisiología, Facultad de Medicina, Universidad de Cádiz, 11003 Cádiz, Spain, and 2Laboratorio de Neurociencia, \\ Facultad de Biología, Universidad de Sevilla, 41012 Sevilla, Spain
}

Nitric oxide (NO) production by neurons in the prepositus hypoglossi $(\mathrm{PH})$ nucleus is necessary for the normal performance of eye movements in alert animals. In this study, the mechanism(s) of action of $\mathrm{NO}$ in the oculomotor system has been investigated. Spontaneous and vestibularly induced eye movements were recorded in alert cats before and after microinjections in the $\mathrm{PH}$ nucleus of drugs affecting the NO-cGMP pathway. The cellular sources and targets of NO were also studied by immunohistochemical detection of neuronal NO synthase (NOS) and NO-sensitive guanylyl cyclase, respectively. Injections of NOS inhibitors produced alterations of eye velocity, but not of eye position, for both spontaneous and vestibularly induced eye movements, suggesting that $\mathrm{NO}$ produced by $\mathrm{PH}$ neurons is involved in the processing of velocity signals but not in the eye position generation. The effect of neuronal NO is probably exerted on a rich CGMP-producing neuropil dorsal to the nitrergic somas in the PH nucleus. On the other hand, local injections of NO donors or 8-Br-cGMP produced alterations of eye velocity during both spontaneous eye movements and vestibulo-ocular reflex (VOR), as well as changes in eye position generation exclusively during spontaneous eye movements. The target of this additional effect of exogenous NO is probably a well defined group of NO-sensitive CGMP-producing neurons located between the $\mathrm{PH}$ and the medial vestibular nuclei. These cells could be involved in the generation of eye position signals during spontaneous eye movements but not during the VOR.

Key words: eye movements; nitrergic neurons; nitric oxide; oculomotor integrator; prepositus hypoglossi nucleus; soluble guanylyl cyclase
We have reported recently that a balanced production of nitric oxide (NO) in the prepositus hypoglossi (PH) nucleus is necessary for the correct performance of spontaneous eye movements in the alert cat (M oreno-L ópez et al., 1996). A $n$ imbalance in the $\mathrm{NO}$ concentration between the two PH nuclei, induced by unilateral injections of NO synthase (NOS) inhibitors or NO donors, resulted in nystagmic eye movements whose slow phases were directed toward the side in which $\mathrm{NO}$ concentration was higher. In the present work, we have used the cat oculomotor system as a model to investigate the possible mechanisms of action and targets of $\mathrm{NO}$ in sensorimotor processing.

The functions of eye movements are to direct the highest vi sual acuity portion of the retina to the objects of interest and to maintain the stability of the visual targets on the retina, despite displacements of the head or the visual surroundings. To perform these functions, motoneurons in the oculomotor nuclei of the brainstem send two types of commands to the extraocular muscles: a velocity signal and a position signal (Fuchs and L uschei, 1970; Skavenski and R obinson, 1973; D elgado-G arcía et al., 1986; De la Cruz et al., 1990).

O cular motoneurons are controlled by several premotor struc-

\footnotetext{
Received A ug. 3, 1998; revised Sept. 28, 1998; accepted Sept. 30, 1998.

This work was supported by Fondo de Investigación Sanitaria G rants 94/0388 and 97/2054, Comunidad Autonoma de Madrid Grant 08.5/0019/1997, and Dirección G eneral de Investigación C ientífica y Technológica G rant PB 93-1175. We thank D r. de $V$ ente for kindly providing the anti-cG M P antibody, R ut G onzález for excellent technical assistance, D r. D elgado-G arcía for his generous help, and D r. Elena $\mathrm{G}$ alea for critical discussion.

Correspondence should be addressed to Dr. C armen Estrada, Á rea de Fisiología, Facultad de M edicina, U niversidad de C ádiz, Plaza F ragela s/n, 11003 C ádiz, Spain. Copyright @ 1998 Society for Neuroscience 0270-6474/98/1810672-08\$05.00/0
}

tures in which the different types of eye movements are generated. Thus, neurons in the pontine reticular formation encode velocity signals during saccades (H ikosaka et al., 1978; K aneko et al., 1981; Strassman et al., 1986a,b), whereas neurons in the medial vestibular ( $\mathrm{M} \mathrm{V}$ ) nucleus fire at a rate related to head velocity during vestibular stimulation (Baker et al., 1969; H ikosaka et al., 1980; M cC rea et al., 1980, 1987; Berthoz et al., 1989; Escudero et al., 1992). Robinson (1968, 1975) proposed that position signals for any kind of eye movement result from the temporal integration (in the mathematical sense) of velocity signals, establishing the concept of the neural integrator. A Ithough the sources of position signals are not completely determined, the $\mathrm{PH}$ nucleus has been identified as one of the structures responsible for neural integration for horizontal eye movements ( $L$ ópezBarneo et al., 1982; C heron et al., 1986b; C annon and R obinson, 1987; Cheron and Godaux, 1987; Delgado-García et al., 1989; E scudero et al., 1992; M cF arland and F uchs, 1992; M ettens et al., 1994; for review, see Fukushima et al., 1992).

The $\mathrm{PH}$ nucleus is a long and narrow nucleus located immediately below the floor of the fourth ventricle. A large number of neurons in this nucleus express NOS, as demonstrated by immunocytochemical techniques (M oreno-L ópez et al., 1996). The PH nucleus receives afferents from different premotor structures, such as the $\mathrm{MV}$ nuclei, the pontine reticular formation, and the contralateral PH nucleus, and sends efferents to these same structures and to the oculomotor nuclei, including the abducens nucleus (McCrea and Baker, 1985) in which motoneurons and internuclear neurons controlling horizontal eye movements are located.

Pharmacological modification of $\mathrm{NO}$ concentration in the $\mathrm{PH}$ 


\section{Spontaneous eye movements}

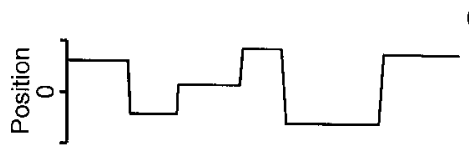

Control

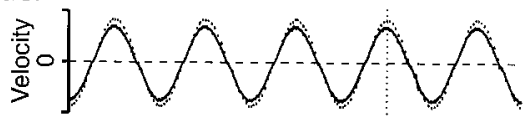

Velocity imbalance
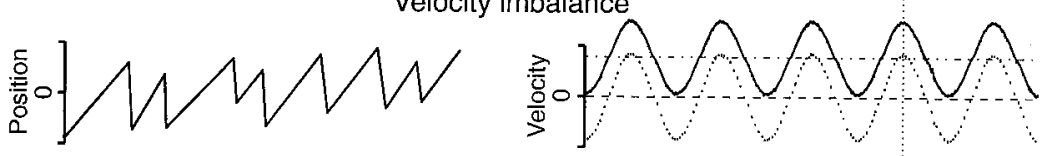

Integrator failure

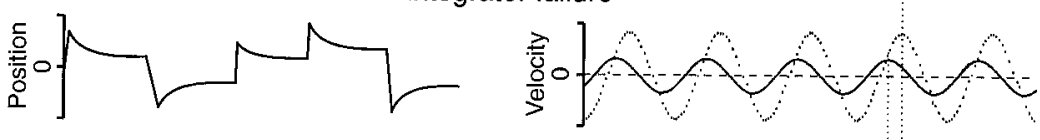

Integrator failure + Velocity imbalance
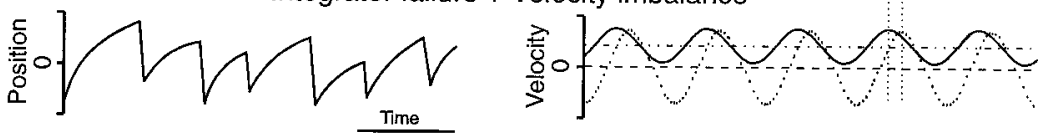

Figure 1. Schematic drawing representing eye movements under normal and altered conditions. Eye position ( position) in spontaneous eye movements (left) and eye velocity (velocity) during VOR induction (right) are schematically represented in a control situation and under hypothetical alterations of velocity processing (velocity imbalance), integration (integrator failure), or both. $\mathrm{O}$ the right, the solid line represents eye velocity, and the dotted line represents head velocity. Because eyes move in a direction opposite to the head during the reflex response, the head velocity curve has been inverted to facilitate comparison with the eye velocity curve. The horizontal dashed line corresponds to the mean head velocity, which by definition is zero. The horizontal dashed and dotted line represents the mean eye velocity, which is also zero in the absence of velocity imbalance. The vertical dotted lines indicate the head and eye phase peaks. L eft, D uring spontaneous movements, alterations in the processing of velocity signals produce nystagmic eye movements with ramp-like slow phases, whereas a failure of oculomotor integration produces gaze-holding impairment, causing exponential drifts toward a central position. When integration is absent, the drift time constant is estimated to be $0.16 \mathrm{sec}$ ( $\mathrm{G}$ oldberg, 1980). If both velocity and integration deficits occur at the same time, a nystagmus with curved slow phases will appear. Right, D uring VOR induction, the velocity imbalance appears as a positive or negative value of the mean slow eye velocity, whereas the integration deficit results in a decreased reflex gain and increased phase lead. A velocity imbalance with gain reduction and enhanced phase lead should be expected when both alterations are present.

nucleus may affect inputs from premotor structures conveying velocity information; additionally or alternatively, such modification may affect intranuclear neurons involved in the velocity-toposition integration. Depending on the target, velocity imbalance and/or integration deficit will occur, leading to well characterized abnormal eye movements ( $C$ annon and R obinson, 1987; G odaux et al., 1993; M ettens et al., 1994; Pastor et al., 1994; G odaux and Cheron, 1996), which are schematically represented in Figure 1. $\mathrm{E}$ ach $\mathrm{PH}$ nucleus simultaneously receives excitatory and inhibitory signals from the contralateral and ipsilateral $\mathrm{M} \mathrm{V}$ nuclei, respectively (Baker and Berthoz, 1975). Because $M V$ nucleus neurons projecting to the $\mathrm{PH}$ nucleus display a tonic discharge (M cCrea et al., 1980; Berthoz et al., 1989), a modification in the transmission of one of these signals would result in a velocity imbalance, as represented in Figure 1, left and right, for spontaneous eye movements and vestibularly induced eye movements, respectively. On the other hand, a failure in the eye position generation should eliminate or decrease the eye position input to the motoneurons, and the eye movements would reflect the eye velocity commands. The consequence is that, during spontaneous eye movements, the eye is unable to maintain an eccentric position after a saccade and exponentially returns to a central position (Fig. 1, left) with a time constant that is inversely proportional to the degree of integrator failure and is finally imposed by the viscoelastic forces acting on the eye in the orbit. During the vestibulo-ocular reflex (VOR), impairment of the velocity-toposition integrator results in a decreased reflex gain and enhanced phase lead, as schematically represented in F igure 1, right.

In the present study, we have analyzed whether the NO produced by nitrergic neurons in the $\mathrm{PH}$ nucleus is involved in the processing of velocity signals, position signals, or both, during either spontaneous eye movements or V OR. W e have determined also the possible targets of $\mathrm{NO}$ by immunostaining of $C G M P$, the second messenger that is activated by $\mathrm{NO}$. We found that $\mathrm{NO}$ produced by $\mathrm{PH}$ neurons participates in the processing of pure velocity signals, probably by interacting with CGM P-containing neuropil within this nucleus. We have also identified a group of $\mathrm{NO}$-sensitive neurons, lateral to the $\mathrm{PH}$ nucleus, that may control the generation of position signals during spontaneous eye movements but not during VOR.

\section{MATERIALS AND METHODS}

Subjects. Fourteen adult cats $(2.5-3.5 \mathrm{~kg})$ of European and A byssinian strains were obtained from an authorized supplier (IFFA Credo, Arbresle, France) and were used as experimental subjects. Experiments were performed in accordance with the European U nion directive 609/ 86/CEE and with Spanish legislation (RD 233/89) on the use of laboratory animals in acute and chronic experiments.

Immunohistochemistry. Six cats were perfused through the left ventricle with $4 \%$ paraformaldehyde in $0.1 \mathrm{~m}$ phosphate buffer, $\mathrm{pH}$ 7.4. A fter the brains were removed, the brainstems were post-fixed for $2 \mathrm{hr}$ and then cryoprotected by incubation for $2 \mathrm{~d}$ with $30 \%$ sucrose at $4^{\circ} \mathrm{C}$. Coronal 40 $\mu \mathrm{m}$ sections were obtained with a freezing microtome. To visualize neurons containing $\mathrm{NO}$-sensitive guanylyl cyclase, the procedure described by Southam and Garthwaite (1993) was used with some modifications. Briefly, two of the animals were perfused through the left ventricle with a physiological solution (in $\mathrm{mm}$ : $120 \mathrm{~N} \mathrm{aCl}, 2 \mathrm{~K} \mathrm{Cl}, 2 \mathrm{CaCl}_{2}$, $26 \mathrm{NaHCO}_{3}, 1.2 \mathrm{~K} \mathrm{H}_{2} \mathrm{PO}_{4}, 1.2 \mathrm{M} \mathrm{gSO}_{4}$, and 11 glucose) at $37^{\circ} \mathrm{C}$ and bubbled with $95 \% \mathrm{O}_{2}$ and $5 \% \mathrm{CO}_{2}$, containing the $\mathrm{NO}$ donor sodium nitroprusside (SNP) (10 mM) and the phosphodiesterase inhibitor 3-isobutylmethylxantine (I BM X) (1 mM ), for $5 \mathrm{~min}$ at $200 \mathrm{ml} / \mathrm{min}$. T wo control animals were treated under the same conditions but in the absence of SN P. A fterward, these cats were perfused with $4 \%$ paraformaldehyde, and the tissue was processed as described above.

NOS immunohistochemistry was performed as described previously (M oreno-L ópez et al., 1996), except that a polyclonal antibody raised against a $22.3 \mathrm{kD}$ a protein fragment of human neuronal NOS (Transduction Laboratories, L exington, $K Y$ ) was used. Tissue sections were processed according to the avidin-biotin peroxidase complex procedure, using an A BC kit (V ector L aboratories, Burlingame, CA). No immunostaining was observed when the primary antibody was omitted.

To visualize neurons containing $\mathrm{NO}$-sensitive soluble guanylyl cyclase, an antibody raised against a CGM P-paraformaldehyde-bovine thyroglobulin complex (Tanaka et al., 1997), kindly provided by D r. de V ente (R ijksuniversiteit Limburg, M aastrich, Netherlands), was used. B efore fixation, the animal was perfused with SN $P$ to activate soluble guanylyl cyclase. No significant labeling was found when staining was performed under the same conditions in animals perfused with I BM $X$ but without SN P. D etails on this staining and the distribution of CG M P-containing neurons in oculomotor nuclei will be given elsewhere (B. M oreno-L ópez, M. Escudero, J. de V ente, and C. Estrada, unpublished data). D rug injection sites were identified in some animals after completion of the recording sessions by injections of either horseradish peroxidase (B oehr- 
inger M annheim, Indianapolis, I N) or biotin dextran amine (M olecular Probes, Eugene, OR) as described previously (Moreno-L ópez et al., 1996).

Physiological experiments. Eight female cats were prepared for chronic recording of eye movements and for microinjection of pharmacological substances into the PH nucleus as described previously ( $M$ oreno-L ópez et al., 1996). B riefly, under general anesthesia (N embutal, $35 \mathrm{mg} / \mathrm{kg}$, i.p.), the cats were implanted bilaterally with Teflon-coated stainless steel coils sutured to the scleral margin of the eye (Fuchs and Robinson, 1966). In the same surgical act, a $4 \times 4 \mathrm{~mm}$ hole was drilled through the occipital bone to allow access to the posterior brainstem via the cerebellum. Bipolar silver stimulating electrodes were implanted bilaterally on the sixth nerve at its exit from the brainstem (stereotaxic coordinates, lateral 3.5 and posterior 1 , according to B erman, 1968). The final location of the stimulating electrode was adjusted to evoke the maximum abducting eye movement with the minimum electrical stimulation ( $50 \mu \mathrm{sec}$, cathodic square pulses of $<0.1 \mathrm{~mA}$ of current intensity). A head-holding system, consisting of three bolts cemented to the skull perpendicular to the stereotaxic plane, was also implanted. Eye coils and stimulating electrodes were connected to a socket attached to the holding system. Field potential and unitary activity were recorded with glass micropipettes of 2- $6 \mathrm{M} \Omega$ of electrode resistance. Further details of this type of chronic preparation have been reported elsewhere (D elgado-G arcía et al., 1986; Escudero et al., 1992).

O ne to 2 weeks later, when there was total recovery from surgery, experiments were performed in the alert cat once every 2-4 d, for 2-3 $\mathrm{hr} / \mathrm{d}$, for a maximum of 4- 8 weeks. D uring the experimental sessions, the animal was lightly restrained by elastic bandages, and the head was fixed $\left(21^{\circ}\right.$, nose down) to the recording table by means of the head-holding system. A glass micropipette was advanced through the cerebellum toward either the left or right abducens nucleus, which was identified by the recording of the antidromic field potential induced by electric stimulation of the ipsilateral sixth nerve. The PH nucleus was found in the same parasagittal plane and posterior to the abducens nucleus, just below the floor of the fourth ventricle. All injections were restricted to the rostral third of the PH nucleus. The correct position of the micropipette was confirmed by recording the characteristic firing discharge of $\mathrm{PH}$ neurons during spontaneous and vestibularly induced eye movements (E scudero et al., 1992). The horizontal V OR was elicited by sinusoidal rotation around the vertical axis at 0.1 and $1 \mathrm{~Hz}$. The amplitude of the table movement was adjusted to keep maximal angular head velocity constant at $30 \% \mathrm{sec}$ for both frequencies. Injections were performed by means of glass micropipettes with tip diameters of 7- $8 \mu \mathrm{m}$, filled with the corresponding drug dissolved in $0.1 \mathrm{M}$ phosphate buffer, $\mathrm{pH}$ 7.4. A ir pulses $\left(1 \mathrm{~kg} / \mathrm{cm}^{2}, 1 \mathrm{sec}\right)$ were applied with an air pressure device connected to the injection micropipette to deliver $40-45 \mathrm{nl} /$ pulse. Spontaneous eye movements were continuously recorded in complete darkness and occasionally in light, with the micropipette in place both before and after injections. E ye movements were calibrated at the beginning of each experimental session by rotating $\left( \pm 10^{\circ}\right)$ the magnetic field frame about both the horizontal and vertical planes. Eye movements during VOR were recorded in the dark both before and after drug injections. Field and unitary electrical activities and head and eye position were stored in an eight-channel video tape recording system and fed into a computer for off-line analysis. E ye and head position signals were sampled at $500 \mathrm{~Hz}$.

A nal ysis of the data. D uring spontaneous eye movements in darkness, the alterations induced by drug injections in the $\mathrm{PH}$ nucleus consisted of nystagmic eye movements with straight or curved slow phases separated by quick resetting movements. A nalysis of the slow phases was performed during the $3 \mathrm{~min}$ period of maximum effect for each injection and immediately before vestibular stimulation. Slow phases with duration greater than $0.5 \mathrm{sec}$ were fit separately by the least squares method to linear and exponential equations and were considered to be linear or exponential when $>80 \%$ of the analyzed phases had a correlation coefficient $>0.99$ or $>0.90$, respectively. Because linear and curved slow phases are indicative of two different alterations (velocity imbalance and eye position generation, respectively) that could be simultaneously induced, the time constants of the exponential slow phases were calculated from the first derivative of eye position (eye velocity) to prevent value underestimation attributable to possible concomitant linear phases in the eye position recording.

D uring VOR, the eye movement response was defined by three parameters: velocity imbalance, reflex gain, and phase lead. The velocity imbalance was measured as the mean slow eye velocity and was expressed
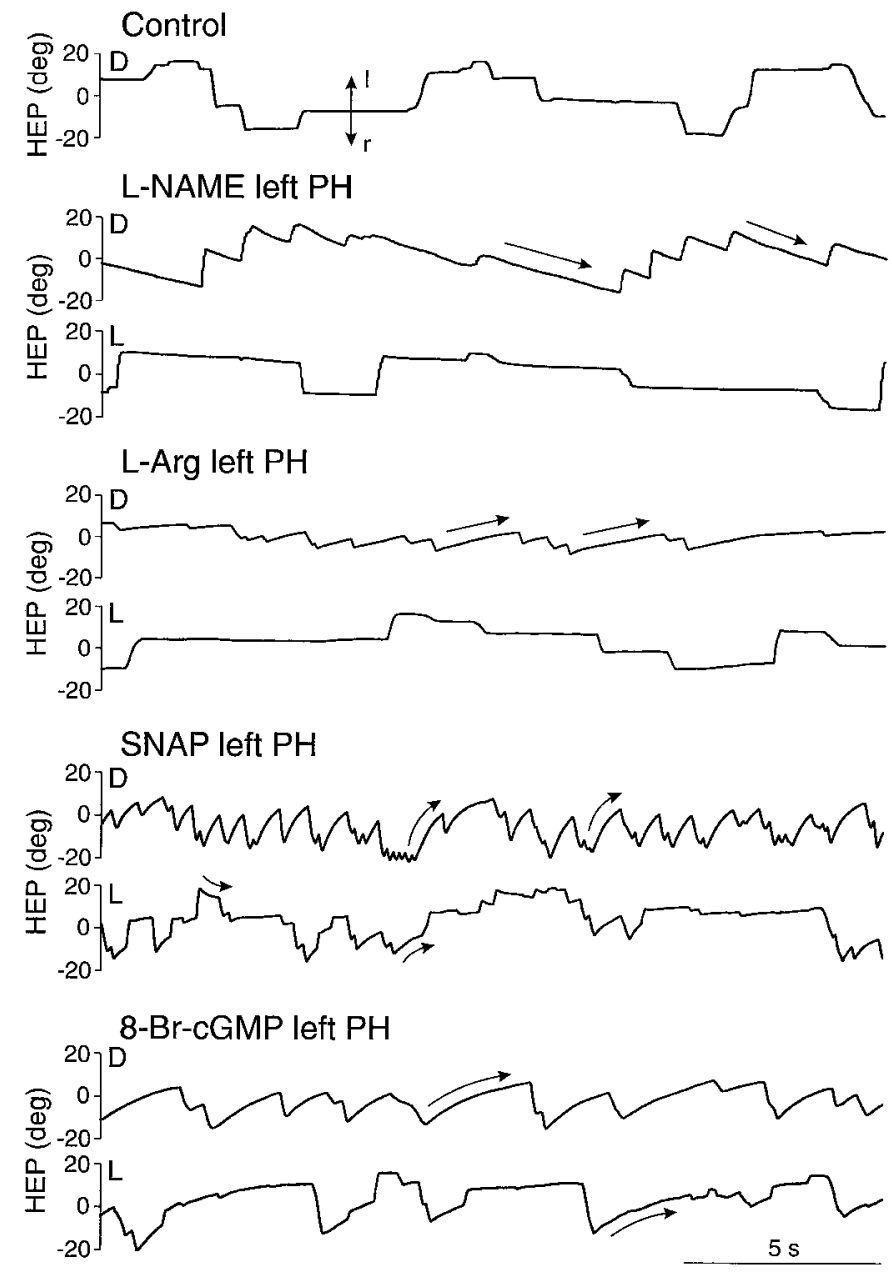

Figure 2. Effects of unilateral injections of drugs affecting the NOCG M P pathway in the PH nucleus of an alert cat during spontaneous eye movements. Recordings of right eye position in the horizontal plane (HEP) under control conditions and after injections of the indicated drugs in the left PH nucleus, in either darkness (D) or light (L). D oses and times after injection were as follows: L-NA M E, $50 \mathrm{nmol}, 19$ (D) and 20 (L) min; L-arginine (L-Arg), $100 \mathrm{nmol}, 8$ (D) and 11 (L) min; SN A P, $20 \mathrm{nmol}, 5$ (D) and 4 (L) min; and 8-Br-cG M P, 4 nmol, 6 (D) and 8 (L) min. Eye position is plotted as degrees (deg). Vertical arrows indicate movement direction: I, left; $r$, right. Straight and curved arrows indicate linear and exponential slow phases, respectively. Slow phases for L-NAM E and L-arginine were best fit to a linear equation, whereas slow phases for SNAP and 8-Br-cGMP were best fit to an exponential equation.

in degrees per second. The reflex gain was calculated as the ratio between the peak-to-peak amplitude of slow eye velocity versus the peak-to-peak amplitude of head velocity. To analyze the head and slow eye velocity, a computer program was developed. For each cycle, the sinusoidal function of the head velocity was calculated by fitting a periodic function (trigonometric polynomial) by the least squares method (Batschelet, 1981). This sine wave was adjusted by cursors to the eye velocity signal. The points of the eye velocity signal, which were in the range of $\pm 10 \%$ with respect to the reference sine wave, were selected, and the rest, corresponding to the quick phases, were ignored. Parameters of the resulting sine wave were calculated as indicated for head velocity. The phase lead was quantified as the temporal shift between the eye and the head position for each hemicycle and then averaged for each complete cycle. This method avoids the shift produced in the eye position by the velocity imbalance, which is of the same magnitude and opposite sign for each hemicycle. D ata from phase shifts were expressed in degrees. Each parameter was measured for at least 10 cycles at $0.1 \mathrm{~Hz}$ and 30 cycles at $1 \mathrm{~Hz}$. 
A


8-Br-cGMP right $\mathrm{PH}$

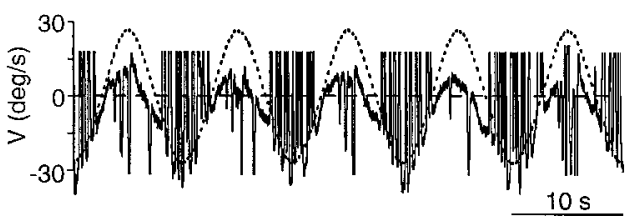

B

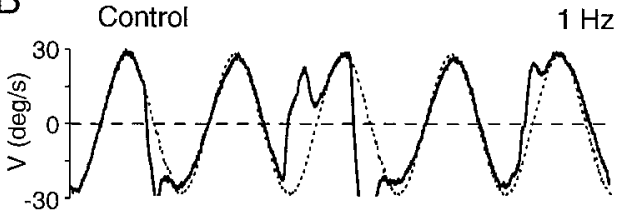

L-NAME right $\mathrm{PH}$
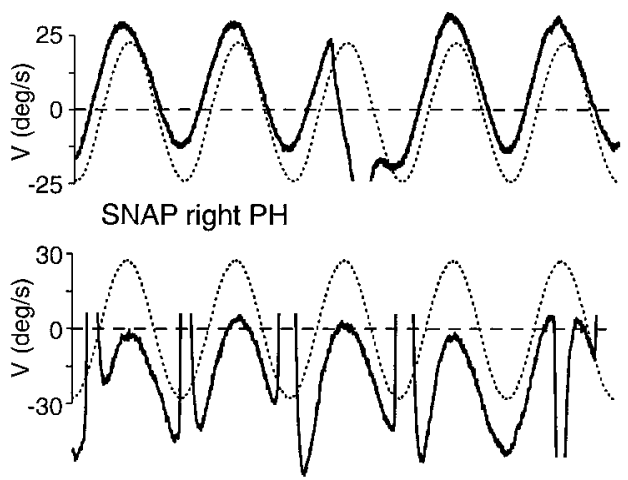

8-Br-cGMP right $\mathrm{PH}$

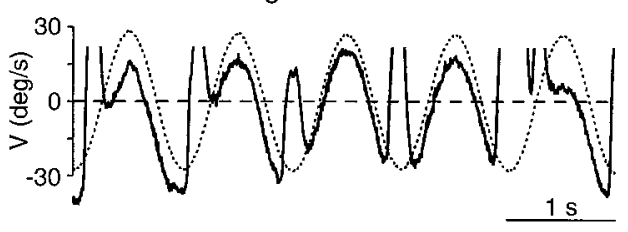

Figure 3. Effects of unilateral injections of drugs affecting the NO-CGM P pathway in the $\mathrm{PH}$ nucleus of an alert cat during sinusoidal vestibular stimulation in darkness. R epresentative recordings of eye (solid line) and head (dotted line) velocity ( $V$ ) during $V O R$ induction by turntable rotation at $0.1(A)$ and $1 \mathrm{~Hz}$ (B) under control conditions and after injections of the indicated drugs in the PH nucleus. The head velocity curve has been inverted to facilitate comparison with the eye velocity curve. M ovement direction as indicated in Figure 2.
R esults are presented as mean \pm SE M, except for normalized control values and when only two experiments were averaged, in which case, mean $\pm S D$ is given. Comparisons within one experiment (for example, when $V O R$ gains were compared in a large number of cycles before and after drug injection) were performed using the Student's t test. Comparisons between two groups of experiments were performed by the nonparametric $M$ ann-W hitney $U$ test. A probability $<0.05$ was considered significant.

\section{RESULTS}

\section{Effect of NOS inhibitors and substrate}

$L$ ocal inhibition of NOS by injections of either L-nitro-arginine methyl ester (L-N A M E ) (10-50 nmol) (Fig. 2) or N-monomethyl$\mathrm{L}$-arginine (30-100 nmol) in the $\mathrm{PH}$ nucleus induced a nystagmus with slow phases directed contralaterally to the injected site, in agreement with our previously reported results (M oreno-L ópez et al., 1996). When the NOS substrate L-arginine (10-100 nmol) was injected in the $\mathrm{PH}$ nucleus, a mild nystagmus toward the ipsilateral side was observed (Fig. 2). In all cases (Fig. 2), the slow phases of the nystagmus were ramp-like with a best fit to a linear equation $(r>0.99)$. When visual information was presented under light conditions, the nystagmus was considerably reduced, and exponential postsaccadic drifts indicative of a loss of position signal were not observed (Fig. 2). These results indicate that modifications of $\mathrm{NO}$ production in the $\mathrm{PH}$ nucleus induce a velocity imbalance without apparent changes in the generation of the eye position signal during spontaneous eye movements.

The NOS inhibitors and L-arginine also modified the oculomotor response during $V O R$ at 0.1 and $1 \mathrm{~Hz}$. A typical recording of the effect of L-NAM E is shown in Figure 3, A and B. Quantification of the data indicated that there was a velocity imbalance (Fig. 4A) without alteration of the reflex gain (Fig. 4B) or the phase lead (Fig. $4 \mathrm{C}$ ) of the reflex. The velocity imbalance was directed to the contralateral side when $\mathrm{PH}$ nucleus NOS was inhibited and toward the ipsilateral side after injection of L-arginine (Fig. 4A). Therefore, as for spontaneous eye movements, unilateral changes in NOS activity in the PH nucleus resulted in abnormal vestibularly induced eye movements, whose exclusi ve alteration was a velocity imbalance without modification of the velocity-to-position integrator.

\section{Effect of NO donors}

Unilateral injections of the NO donors S-nitroso-Nacetylpenicillamine (SN A P) (5-20 nmol) or SN P (5-35 nmol) in the $\mathrm{PH}$ nucleus produced nystagmic eye movements with slow phases toward the ipsilateral side as reported previously (M oreno-L ópez et al., 1996). In contrast to the effects of NOS modulators, the slow phases were curved (Fig. 2) and were best fit by an exponential. The mean time constants of the adjusted exponential during the maximum effects of SN A P and SN P were $0.56 \pm 0.03$ and $0.78 \pm 0.23 \mathrm{sec}$ (mean $\pm \mathrm{SEM} ; n=3$ ), respectively. When the nystagmus was attenuated under light conditions, horizontal saccades were followed by centripetal exponential drifts (Fig. 2). These results reveal a velocity imbalance combined with a gaze-holding deficit for horizontal spontaneous eye movements. D uring $\mathrm{VOR}$, unilateral $\mathrm{NO}$ donor injections in the $\mathrm{PH}$ nucleus also produced a velocity imbalance (Figs. 3A, B, 4A). However, the gain and phase lead of the reflex remained intact (Figs. 3A, B, 4B,C), indicating no disruption of the velocity-toposition integration during $\vee O R$, despite the alterations observed during spontaneous eye movements both before and after the VOR induction period. Table 1 shows the mean variation values of gain and phase lead during VOR for individual injections, together with the mean time constant of the postsaccadic drift during spontaneous eye movements measured immediately before vestibular stimulation. 

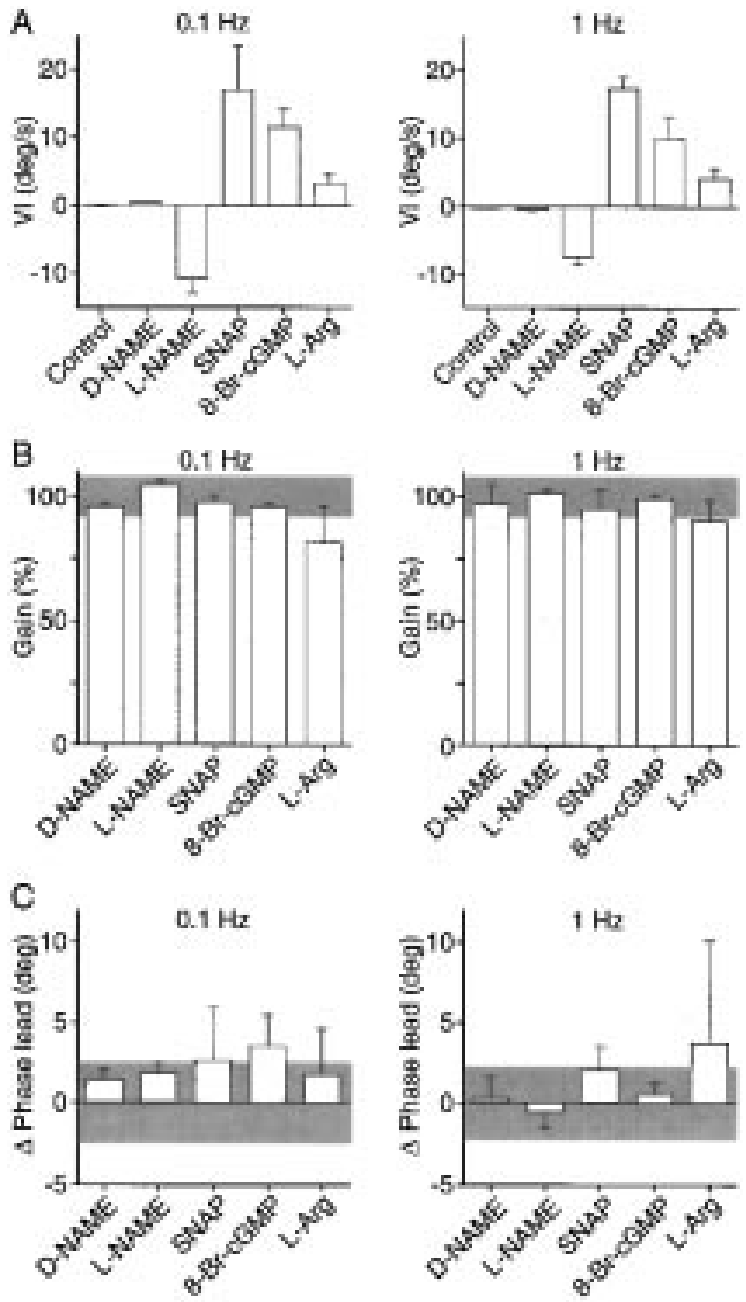

Figure 4. Velocity imbalance, gain, and phase lead of the vestibuloocular reflex after local administration in the PH nucleus of drugs affecting the NO-cG M P pathway. A, V elocity imbalance (VI) produced by the different drugs during $V O R$ induction by rotation of the head at 0.1 and $1 \mathrm{H}$ z. Positive values represent velocity imbalance toward the injected side and negative values toward the contralateral side. B, Gain of the VOR induced by rotation of the head at 0.1 and $1 \mathrm{~Hz}$ after injection of the different drugs. $\mathrm{G}$ ain is expressed as a percentage of the control value for each experiment. Gray bar, $100 \% \pm$ SD of control values. C, Changes in phase lead during the VOR induced by rotation of the head at 0.1 and 1 $\mathrm{Hz}$ produced by injection of the different drugs. Values are expressed in degrees (deg). G ray bar, $0 \pm S D$ of control values. $n=2$ for $D-N A M E ; n=$ 3 for SNAP and L-arginine; $n=4$ for 8 -Br-CGMP; and $n=5$ for L-NAME.

\section{Effect of cGMP analogs}

$L$ ocal injections of the permeant CGM P analog 8-Br-cGM P (4-10 nmol) in the PH nucleus induced a nystagmus whose slow phases, directed ipsilaterally to the injected side, were best fit to an exponential equation with a mean time constant of $0.92 \pm 0.10$ $\sec$ (mean $\pm \mathrm{SEM} ; \mathrm{n}=4$ ) during the period of maximum effect. Under light conditions, a loss of eye position signal was also observed (Fig. 2). D uring V O R , 8-B r-cG M P produced a velocity imbalance (Figs. 3A, $B, 4 A$ ) without alteration of the reflex gain and phase lead ( $F$ igs. 3A, B, 4B, C). Therefore, the CG M P analog behaved exactly like the $\mathrm{NO}$ donors, suggesting that the effects of $\mathrm{NO}$ on eye velocity during spontaneous movements and VOR and on eye position during spontaneous eye movements were mediated by the activation of soluble guanylyl cyclase.
Table 1. E ffect of individual drug injections on VOR gain, phase lead, and postsaccadic drift time constant

\begin{tabular}{|c|c|c|c|c|c|}
\hline \multirow[b]{2}{*}{ Drug } & \multicolumn{2}{|c|}{ VOR $(0.1 \mathrm{~Hz})$} & \multicolumn{2}{|c|}{ VOR (1 Hz) } & $\begin{array}{l}\text { Spontaneous eye } \\
\text { movements }\end{array}$ \\
\hline & $\Delta G$ ain $^{a}$ & $\begin{array}{l}\Delta \text { Phase } \\
\text { lead } \\
\text { (deg) }^{\text {b }}\end{array}$ & $\Delta \mathrm{G}$ ain $^{\mathrm{a}}$ & $\begin{array}{l}\Delta \text { Phase } \\
\text { lead } \\
\text { (deg) }^{\text {b }}\end{array}$ & $\begin{array}{l}\text { Time constant } \\
(\mathrm{sec})^{\mathrm{c}}\end{array}$ \\
\hline \multirow[t]{3}{*}{ SNAP } & -0.01 & -2.0 & -0.03 & 2.0 & $0.90 \pm 0.40$ \\
\hline & 0.02 & 0.9 & -0.12 & 4.6 & $0.57 \pm 0.12$ \\
\hline & -0.06 & $8.9 *$ & 0.07 & -0.5 & $0.73 \pm 0.26$ \\
\hline \multirow[t]{4}{*}{ 8-Br-cG M P } & -0.03 & 7.0 & 0.00 & 2.6 & $1.78 \pm 0.44$ \\
\hline & -0.01 & 6.5 & -0.03 & -0.8 & $1.45 \pm 0.76$ \\
\hline & -0.03 & 1.1 & 0.03 & -0.1 & $1.70 \pm 0.24$ \\
\hline & -0.07 & -0.8 & -0.02 & 0.3 & $0.84 \pm 0.37$ \\
\hline
\end{tabular}

${ }^{a} \mathrm{~V}$ alues represent changes in V OR gain after drug injection. A bsolute gain values in control conditions were $0.83 \pm 0.08$ for $0.1 \mathrm{~Hz}$ and $0.94 \pm 0.12$ for $1 \mathrm{~Hz} . \mathrm{n}=7$.

${ }^{b} \mathrm{~V}$ alues represent changes in V OR phase lead after drug injection. A bsolute phase lead values in control conditions were $10.2 \pm 2.8$ for $0.1 \mathrm{~Hz}$ and $6.9 \pm 2.6$ for $1 \mathrm{~Hz}$. $\mathrm{n}=7$.

${ }^{\mathrm{c}} \mathrm{M}$ ean $\pm \mathrm{SD}$ of time constants calculated immediately before VOR induction.

$* p<0.05$ when phase leads before and after injection were compared.

\section{Localization of neurons containing NOS and NO-sensitive guanylyl cyclase}

To find the possible targets of NO, cG M P immunohistochemistry was performed in brainstem sections from cats perfused with SN P before fixation. U sing this technique in combination with NOS immunohistochemistry in similar sections, we found that in the $\mathrm{PH}$ nucleus NOS was present in a group of densely packed neurons (Fig. 5A), whereas CG M P was present in a rich neuropil in the dorsal part of the nucleus (Fig. 5B). No CGM Pimmunoreactive ( $C G M P$-ir) cell bodies were found in the $\mathrm{PH}$ nucleus. In addition, a cluster of NO-sensitive CG M P-ir neuronal cell bodies and neuropil were identified in an intermediate zone between the $\mathrm{PH}$ and $\mathrm{M} \mathrm{V}$ nuclei (Fig. 5B,C). The latter structure was at a distance of $\sim 0.4 \mathrm{~mm}$ from the nitrergic neurons in the $\mathrm{PH}$ nucleus, suggesting that these cells might be the target for the effects of NO donors and 8-Br-cGM P, which were not observed when NOS activity was modified experimentally.

\section{DISCUSSION}

In this study, we have shown that $\mathrm{NO}$ produced in the $\mathrm{PH}$ nucleus participates in the processing of velocity signals in the alert cat, probably by acting on the CG M P-ir neuropil located in the dorsal part of the nucleus. $O \mathrm{n}$ the other hand, local administration of exogenous NO produced, in addition, an alteration in the velocity-to-position integrator for spontaneous eye movements, suggesting the existence of another NO target, which is not reached by endogenous $\mathrm{NO}$ in physiological conditions. A group of neurons, located between the $\mathrm{PH}$ and $\mathrm{M} \mathrm{V}$ nuclei and identified by $\mathrm{CGM}^{\mathrm{P}}$ immunohistochemistry, might be responsible for the integration deficit caused by exogenous NO.

Modification of NOS activity in the $\mathrm{PH}$ nucleus induced a nystagmus during spontaneous eye movements in the dark and a velocity imbalance without alteration of gain or phase lead during VOR. The slow phases of the nystagmic eye movements were ramp-like and, when the animal was returned to conditions of light and the nystagmus was attenuated, no deficits in gazeholding were observed. A II these data indicate that NO produced by $\mathrm{PH}$ nitrergic neurons is not involved in the velocity-to-position 

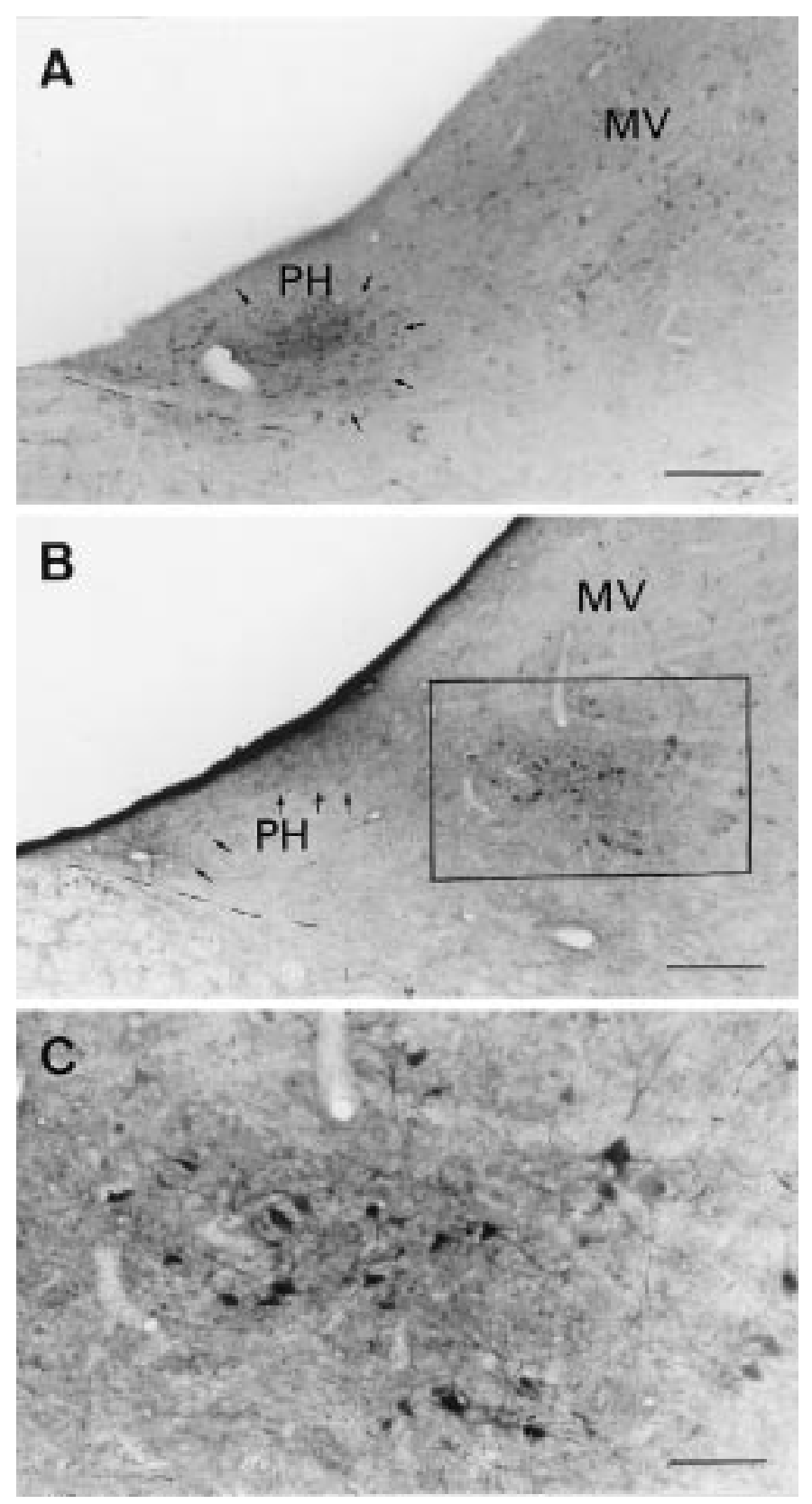

Figure 5. Distribution of nitrergic neurons and neurons containing NOsensitive guanylyl cyclase in the PH nucleus and adjacent areas. A, Photomicrograph of a coronal brainstem section (posterior 8, according to Berman, 1968) through the PH and M V nuclei stained with a polyclonal antibody recognizing neuronal NOS. A dense group of nitrergic neurons is observed in the PH (arrows). B, Photomicrograph of a coronal brainstem section at a similar rostrocaudal level stained with a polyclonal antibody raised against CGM P. The neuropil is densely stained in the dorsal part of the PH nucleus (arrows), and a cluster of labeled cell bodies (boxed area) appears in an intermediate location between the PH and M V nuclei. $C, H$ igher magnification of the area indicated in $B$, showing the morphology of N O-sensitive CG M P-producing neurons. Scale bars: A, B, $250 \mu \mathrm{m} ; \mathrm{C}, 100 \mu \mathrm{m}$.

integration mechanism but only in the processing of pure velocity signals.

With regard to the physiological targets of $\mathrm{NO}$ produced by the $\mathrm{PH}$ neurons, the absence of CG M P-ir cell bodies in the nucleus rules out a possible effect of $\mathrm{NO}$ on intranuclear neurons. $\mathrm{R}$ ather, the discovery of an intense CG M P-ir neuropil suggests that NO is acting on nerve fibers or terminals reaching the $\mathrm{PH}$ nucleus from other structures. This is in agreement with the proposed role of $\mathrm{NO}$ as a retrograde messenger, i.e., NO is being released from the postsynaptic neuron and acting on presynaptic terminals in the C NS (Gally et al., 1990; Schuman and M adison, 1994). The affected terminals cannot originate in the contralateral $\mathrm{PH}$ nucleus because of the absence of CGM P-ir cell bodies. A ccording to the functional results, NO may be acting on terminals of vestibular origin, which convey velocity signals and display a basal discharge in the absence of stimulation by head movements. A Ithough the results are not conclusive, this possibility is supported by our recent finding that both the $\mathrm{MV}$ and inferior vestibular nuclei contain CGM P-immunostained cell bodies (M oreno-L ópez, E scudero, de V ente, and E strada, unpublished observations).

Injections of $\mathrm{NO}$ donors in the $\mathrm{PH}$ nucleus also altered the velocity of spontaneous and vestibularly induced eye movements, but they had an additional effect on the velocity-to-position integration during spontaneous eye movements, as can be deduced from the exponential fit of nystagmic slow phases in darkness and the centripetal drifts that followed saccades under conditions of light. The apparent lack of correspondence between the effects of NOS inhibitors and NO donors can be interpreted by understanding the differences in distribution volumes between endogenous and exogenous NO. A fter cerebral injections, the diffusion volume of the drugs was probably large enough to include the $\mathrm{PH}$ nucleus and some adjacent structures within a few seconds. When $\mathrm{NO}$ donors were injected, this entire volume was exposed to NO. H owever, when an NOS substrate or an NOS substrate analog was injected, they could only affect the activity of NOS, which, within the volume exposed to the injection, is expressed only by the nitrergic cell bodies in the $\mathrm{PH}$ nucleus. A ctivation or inhibition of NOS would modify the endogenous NO production and change $\mathrm{NO}$ concentration in a small volume around the nitrergic neurons. This volume can be estimated as a sphere with a radius of $\sim 100$ $\mu \mathrm{m}$ around the NO point source (W ood and G arthwaite, 1994). Therefore, the $\mathrm{NO}$-sensitive structures responsible for the integration deficit caused by NO donors, but not by NOS inhibitors, should lie within the drug diffusion volume but outside the volume of influence of $\mathrm{NO}$ produced by $\mathrm{PH}$ neurons.

The NO targets responsible for the eye position deficit were probably structures containing soluble guanylyl cyclase, because the same alteration appeared when a CG M P analog was injected. In an attempt to identify the possible targets of NO donors, immunostaining for CGM $P$ was analyzed in brainstem regions close to, but not within, the $\mathrm{PH}$ nucleus. This approach revealed the existence of a small area in an intermediate position between the $\mathrm{PH}$ and $\mathrm{MV}$ nuclei, containing a well defined group of CG M P-ir neurons. This area cannot be defined by cytoarchitectonic criteria using N issl staining (our unpublished observations), nor has it been described previously in the cat by the use of any other morphological marker. A ccording to its location, this group of CGM P-ir neurons may correspond to the marginal zone nucleus that has been characterized by histological and physiological criteria in monkeys. I $f$ the cat marginal zone is the actual target of exogenous $\mathrm{NO}$ in our experiments, these neurons should play a role in the integration process during spontaneous eye movements. This is in agreement with the present knowledge on the primate marginal zone, which is considered a component of the saccadic integrator, because virtually all of its neurons are burstposition neurons ( $\mathrm{M} \mathrm{CF}$ arland and Fuchs, 1992) projecting to the 
abducens nucleus (L anger et al., 1986) and to the $\mathrm{PH}$ nucleus (Belknap and M cCrea, 1988).

It is considered currently that a common integrator is in charge of the different types of eye movements, including the gaze-holding integrator, which maintains eye position after saccades, and the velocity-to-position integrator for the VOR. This hypothesis was initially proposed by R obinson (1975) and has been supported by several experimental findings. Thus, lesions in the $\mathrm{PH}$ and/or $\mathrm{M} \mathrm{V}$ nuclei of cats and monkeys produced simultaneous alterations of integration in the different subsystems tested (Cheron et al., 1986a,b; C annon and Robinson, 1987). Furthermore, Godaux and Cheron (1996) have reported that in alert cats the eye position sensitivity of $\mathrm{PH}$ neurons during intersaccadic fixation was equal to that measured during VOR. However, some pharmacological results suggest that at least some of the subsystems may have separate integrators (Godaux and L aune, 1983; Pastor et al., 1994; Y okota et al., 1994). In our experiments, the use of NO donors, as well as 8-Br-cGM P, revealed an alteration of the integrator for spontaneous eye movements, whereas the V OR gain or phase did not change, indicating that the integrator for VOR remained intact. H ence, the integration processes for at least the two oculomotor subsystems tested occur by different mechanisms, because they can be pharmacologically dissociated.

O ur results also suggest that the $\mathrm{NO}$-sensitive CGM P-ir neurons in the cat marginal zone are part of a gaze-holding mechanism specific for saccades. Similarly, small permanent lesions of the primate marginal zone caused by ibotenic acid impaired the gaze-holding in the horizontal plane but not the integration of signals from vestibular sources (K aneko, 1992, 1997). T he lateral location of this area, $\sim 2 \mathrm{~mm}$ from the midline, may explain why Godaux and Cheron (1996), while exploring an area located 1.2-1.6 $\mathrm{mm}$ from the midline, did not record neurons with position sensitivity specific for postsaccadic fixations.

Three conclusions can be drawn from the present results: (1) $\mathrm{NO}$ produced in the $\mathrm{PH}$ nucleus is involved exclusively in the processing of horizontal eye velocity signals, without participation in the integration process occurring in this nucleus; (2) NO action in the $\mathrm{PH}$ nucleus is effected by a retrograde action on afferent fibers, probably arriving from the vestibular nuclei; and (3) a cluster of CGM P-ir neurons located in the marginal zone between the $\mathrm{PH}$ and the $\mathrm{MV}$ nuclei mediates the eye position signal generation during spontaneous, but not vestibularly induced, eye movements, indicating that there is more than one integrator controlling eye movements.

\section{REFERENCES}

Baker R, Berthoz A (1975) Is the prepositus hypoglossi nucleus the source of another vestibulo-ocular pathway? Brain R es 86:121-127.

Baker R, M ano N, Shimazu H (1969) Postsynaptic potentials in abducens motoneurons induced by vestibular stimulation. B rain R es 15:577580.

Batschelet $E$ (1981) C ircular statistics in biology. L ondon: A cademic.

Belknap DE, M cCrea R A (1988) A natomical connections of the prepositus and abducens nuclei in the squirrel monkey. J Comp Neurol 268:13-28.

Berman A L (1968) The brain stem of the cat: a cytoarchitectonic atlas with stereotaxic coordinates. M adison, W I: U niversity of Wisconsin.

Berthoz A, Droulez J, V idal PP, Y oshida K (1989) Neural correlates of horizontal VOR cancellation during rapid eye movements in the cat. J Physiol (L ond) 419:717-751.

Cannon SC, Robinson DA (1987) L oss of the neural integrator of the oculomotor system from brain stem lesions in monkey. J Neurophysiol 57:1383-1409.
Cheron G, Godaux E (1987) Disabling of the oculomotor neural integrator by kainic acid injections in the prepositus vestibular complex of the cat. J Physiol ( $L$ ond) 394:267-290.

Cheron G, Gillis P, Godaux E (1986a) Lesion in the cat prepositus complex: effects on the optokinetic system. J Physiol ( $L$ ond) 372:95-111.

Cheron G, G odaux E, L aune J M , V anderkelen B (1986b) L esions in the cat prepositus complex: effects on the vestibulo-ocular reflex and saccades. J Physiol (L ond) 372:75-94.

De la Cruz R R, Escudero M, Delgado-García J M (1990) Behavior of medial rectus motoneurons in the alert cat. E ur J N eurosci 1:288-295.

Delgado-García J M , D el Pozo F, Baker R (1986) Behavior of neurons in the abducens nucleus of the alert cat. I. M otoneurons. N euroscience 17:929-952.

Delgado-García J M, Vidal PP, Gómez C, Berthoz A (1989) A neurophysiological study of prepositus hypoglossi neurons projecting to oculomotor and preoculomotor nuclei in the alert cat. Neuroscience 29:291-307.

Escudero M, de la Cruz R R, D elgado-García J M (1992) A physiological study of vestibular and prepositus hypoglossi neurones projecting to the abducens nucleus in the alert cat. J Physiol (L ond) 458:539-560.

Fuchs A F, L uschei ES (1970) Firing patterns of abducens neurons of alert monkeys in relationship to horizontal eye movement. J Neurophysiol 33:382-392.

Fuchs A F, R obinson DA (1966) A method for measuring horizontal and vertical eye movement chronically in the monkey. J Appl Physiol 21:1068-1070.

Fukushima K, K aneko CR S, Fuchs A F (1992) The neuronal substrate of integration in the oculomotor system. Prog Neurobiol 39:609-639.

Gally JA, M ontague PR, R eeke GN, Edelman GM (1990) The NO hypothesis: possible effects of a short lived, rapidly diffusible signal in the development and function of the nervous system. Proc N atl A cad Sci U SA 87:3547-3551.

Godaux E, Cheron G (1996) The hypothesis of the uniqueness of the oculomotor neural integrator: direct experimental evidences in the cat. J Physiol (L ond) 492:517-527.

Godaux E, Laune J M (1983) The saccadic system and the vestibuloocular reflex in the cat do not share the same integrator. N eurosci L ett 38:263-268.

Godaux E, M ettens P, Cheron G (1993) Differential effect of injections of kainic acid into the prepositus and the vestibular nuclei of the cat. J Physiol (L ond) 472:459-482.

Goldberg J (1980) Activity of abducens nucleus units in the alert cat. PhD thesis, U niversity of C alifornia at Berkeley.

Hikosaka O, I gusa Y, Nakao S, Shimazu H (1978) Direct inhibitory synaptic linkage of pontomedullary reticular burst neurons with abducens motoneurons in the cat. Exp Brain Res 33:337-352.

H ikosaka O, Nakao S, Shimazu H (1980) Postsynaptic inhibition underlying spike suppression of secondary vestibular neurons during quick phases of vestibular nystagmus. Neurosci L ett 16:21-26.

K aneko CR S (1992) E ffects of ibotenic acid lesions of nucleus prepositus hypoglossi on optokinetic and vestibular eye movements in the alert, trained monkey. A nn N Y A cad Sci 656:408-427.

K aneko CR S (1997) E ye movement deficits after ibotenic acid lesions of the nucleus prepositus hypoglossi in monkeys. I. Saccades and fixation. J Neurophysiol 78:1753-1768.

K aneko CRS, Evinger C, Fuchs A F (1981) Role of cat pontine burst neurons in generation of saccadic eye movements. J Neurophysiol 46:387- 408.

L anger T, K aneko CR S, Scudder CA, Fuchs A F (1986) A fferents to the abducens nucleus in the monkey and cat. J Comp Neurol 245:379-400.

L ópez-Barneo J , D arlot C, B erthoz A, Baker R (1982) N euronal activity in prepositus nucleus correlated with eye movement in the alert cat. J Neurophysiol 47:329-352.

McCrea R A, Baker R (1985) A natomical connections of the nucleus prepositus of the cat. J Comp Neurol 237:377- 407.

M cCrea R A, Y oshida K, Berthoz A, Baker R (1980) Eye movements related activity and morphology of second order vestibular neurons terminating in the cat abducens nucleus. E xp Brain R es 40:468- 473.

M cCrea R A , Strassman A, M ay E, H ighstein SM (1987) A natomical and physiological characteristics of vestibular neurons mediating the horizontal vestibuloocular reflex in the squirrel monkey. J Comp Neurol 264:547-570.

M CF arland J L, Fuchs A F (1992) D ischarge patterns in nucleus preposi- 
tus hypoglossi and adjacent medial vestibular nucleus during horizontal eye movements in behaving macaques. J Neurophysiol 68:319-332.

$M$ ettens P, G odaux E, C heron G, Galiana H L (1994) E ffect of muscimol microinjection into the prepositus hypoglossi and the medial vestibular nuclei on cat eye movements. J N europhysiol 72:785- 802.

Moreno-L ópez B, Escudero M, D elgado-García J M, Estrada C (1996) $\mathrm{N}$ itric oxide production by brain stem neurons is required for normal performance of eye movements in alert animals. Neuron 17:739-745.

Pastor A M, de la Cruz R R, Baker R (1994) Eye position and eye velocity integrators reside in separate brain stem nuclei. Proc Natl Acad Sci U SA 91:807-811.

Robinson DA (1968) Eye movement control in primates. Science $161: 1219-1224$.

Robinson DA (1975) O culomotor control signals. In: Basic mechanisms of ocular motility and their clinical implications (L ennerstrand $G$, Bach-y-R ita P, eds), pp 337-374. Oxford: Pergamon.

Schuman EM, Madison DV (1994) Nitric oxide and synaptic function. A nnu Rev Neurosci 17:153-183.

Skavenski A A, Robinson DA (1973) Role of abducens neurons in vestibuloocular reflex. J Neurophysiol 36:724-737.
Southam E , G arthwaite J (1993) The nitric oxide-cyclic G M P signalling pathway in the rat brain. Neuropharmacology 32:1267-1277.

Strassman A, H ighstein SM, M cCrea R A (1986a) A natomy and physiology of saccadic burst neurons in the alert squirrel monkey. I. E xcitatory burst neurons. J Comp Neurol 249:337-357.

Strassman A, H ighstein SM, M CCrea R A (1986b) A natomy and physiology of saccadic burst neurons in the alert squirrel monkey. II. Inhibitory burst neurons. J Comp Neurol 249:358-380.

Tanaka J, Markerink-van Ittersum M, Steinbusch H W M, de Vente J (1997) Nitric oxide-mediated CG M P synthesis in oligodendrocytes in the developing rat brain. Glia 19:286-297.

Wood J, Garthwaite J (1994) M odels of the diffusional spread of nitric oxide: implications for neural nitric oxide signaling and its pharmacological properties. Neuropharmacology 33:1235-1244.

Y okota J I, R eisine H, C ohen B (1994) V elocity storage and effects of injection of GA BA ergic substances in the prepositus hypoglossi nuclei. In: Contemporary ocular motor and vestibular research: a tribute to $D$ avid $A$. Robinson. (Fuchs A F, Brandt $T$, B üttner $U, Z$ ee $D$, eds), pp 453-461. Stuttgart, G ermany: G eorg Thieme V erlag. 